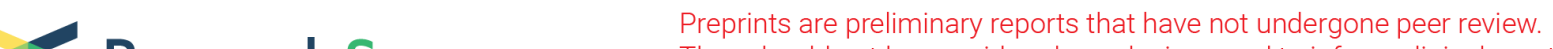 Research Square
They should not be considered conclusive, used to inform clinical practice,
or referenced by the media as validated information.
}

\section{Pregnancy Outcome in Late-onset Severe OHSS Patients Following Different Ascetic Fluid Drainage and Comparison With the Non-OHSS IVF Patients}

\section{Ren YUN}

Centre of Reproductive Medicine, Department of Obstetrics and Gynecology, Peking University, Third Hospital,Beijing 100191, China 2.Key Laboratory of Assisted Reproduction, Ministry of Education, Beijing 100191, China

rong li ( $\nabla$ bysylr@aliyun.com )

Centre of Reproductive Medicine, Department of Obstetrics and Gynecology, Peking University, Third Hospital,Beijing 100191, China 2.Key Laboratory of Assisted Reproduction, Ministry of Education, Beijing 100191, China

\section{Yang Shuo}

Centre of Reproductive Medicine, Department of Obstetrics and Gynecology, Peking University, Third Hospital,Beijing 100191, China 2.Key Laboratory of Assisted Reproduction, Ministry of Education, Beijing 100191, China

\section{Yang Rui}

Centre of Reproductive Medicine, Department of Obstetrics and Gynecology, Peking University, Third Hospital,Beijing 100191, China 2.Key Laboratory of Assisted Reproduction, Ministry of Education, Beijing 100191, China

\section{Song Dong hong}

Centre of Reproductive Medicine, Department of Obstetrics and Gynecology, Peking University, Third Hospital,Beijing 100191, China 2.Key Laboratory of Assisted Reproduction, Ministry of Education, Beijing 100191, China

\section{Qiao Jie}

Centre of Reproductive Medicine, Department of Obstetrics and Gynecology, Peking University, Third Hospital,Beijing 100191, China 2.Key Laboratory of Assisted Reproduction, Ministry of Education, Beijing 100191, China

\section{Research}

Keywords: OHSS, IVF-ET, ascite draining, paracentesis, Central Venous catheter, pregnancy outcome

Posted Date: August 4th, 2020

DOl: https://doi.org/10.21203/rs.3.rs-51728/v1 
License: (c) (i) This work is licensed under a Creative Commons Attribution 4.0 International License. Read Full License 


\section{Abstract}

\section{Introduction区}

Ovarian hyperstimulation syndrome (OHSS) is an iatrogenic serious complication during assisted reproductive technology, the aim of our research is to explore the differences of pregnancy outcome between severe OHSS patients and the non-OHSS IVF patients, and also compare the pregnancy outcome of different ascites drainage way of severe OHSS patients.

\section{Design:}

This is a retrospective cohort analysis carried out in a University-affiliated reproductive center from 2012 to 2019 ,between 359 women with severe OHSS following ascites draining and 345 non-OHSS women (matched by age and retrieved oocyte number).We examined the rates of clinical pregnancy, multiple pregnancies, miscarriage, live birth and preterm delivery between the two groups. Within the OHSS group (central venous catheter group vs paracentesis group), odds ratios (ORs) and 95\% confidence intervals (Cls) of measure of clinical pregnancy were also analyzed.

\section{Result】}

The biochemical pregnancy loss rate of non-OHSS patients was significantly higher than that of severe OHSS patients ( $11.1 \%$ vs $0.6 \%$ ), the live birth, multiple pregnancy (twin pregnancy ) was much more in severe OHSS group than in control group( $88.9 \%$ vs $73.5 \% ; 57.6 \%$ vs $28.1 \%$ respectively), and there was no significant difference between singleton and twin preterm rate and neonatal birth weight between OHSS and control group. Binary logistic regression analysis revealed multiple pregnancy and long-protocol showed relatively high odds ratio, suggest that the

long-protocol of COS and multiple pregnancy are the risk factor of severe OHSS.

Among severe OHSS following ascites draining, in comparison with abdominal paracentesis and central venous catheter, the ascites volume in central venous catheter group was much more than abdominal paracentesis group, there was significant difference between the two groups. There was no significant difference between the two groups in comparison of live birth, and the birth weight of singleton and twin between these two groups. but the premature birth rate of singleton in group 1 was $9.3 \%$ (10/107), whereas in group 2 , it was $3.75 \%$.

\section{Conclusion》}

In conclusion, this preliminary report suggests that for the IVF-ET patients, less basic follicle number, $\mathrm{GnRH}$ antagonist cycle, singleton maybe the protective factors for the late-onset OHSS. Paracentesis and central venous catheter are all the effective modality to manage the ascites for the severe OHSS patients. For the patients who have the possibility of repeated paracentesis, catheter is a safe and effective way. 


\section{Introduction}

Ovarian hyperstimulation syndrome (OHSS) is an iatrogenic serious complication of controlled ovarian stimulation during assisted reproductive technology, with overall incidence of $1 \%$ for the severe stage, OHSS is usually self-limited, but sometimes life-threatening. Among the serious manifestations of OHSS are ascites and pleural effusion. [1]

The pathophysiology of ascites and pleural effusion in OHSS patients is an increase in vascular permeability followed by extravasation of intravascular fluid into the third space [2]. The triggering mechanism is the increase in vascular endothelial growth factor (VEGF) mediated by hCG. The source of HCG includes exogenous hCG and endogenous hCG produced by a successfully implanted embryo. The former is called 'early OHSS' and the latter 'late OHSS'[3].

The classification of OHSS was defined according to the criteria of Golan et al. [4] and Navot et al[5]. Massive ascites were one of evaluation criterion of severe OHSS, it results in increasing abdominal pressure, decreasing venous returns, compromising cardiac output and renal perfusion and the following deteriorating kidney functions.[5]

For the patients that develop into severe OHSS with ascites, paracentesis is a frequently used way of treatment, prompt draining fluid produces significant clinical and biochemical improvement, following abdominal paracentesis, decreased intra-abdominal pressure has been shown to improve the renal perfusion, leading to spontaneous diuresis, increase the cardiac output, avoid serous sequelae of haemoconcentration, hypotension, severe respiratory compromise and hastening the resolution of the process $([6,7][8])$.because the late forms of severe OHSS often have symptom about 8 days after embryo transfer, and last more than one week, in critical cases of OHSS, multiple paracentesis may be required for drainage to relieve symptoms, and repeated abdominal paracentesis increases the pain of patients, and the risk of viscera injuries, the placement of a central venous catheter instead of multiple needle paracentesis would permit drainage through one rather than several interventions, avoid the need for repeated aspiration procedure and the central venous catheter can last for about one week, and because most of the severe OHSS clinical manifestations are self-limited, one week is enough for most of the severe OHSS patients to heal.

The objective of this study was to compare the difference of late-onset OHSS with severe ascites and non-OHSS IVF-ET patients in COS (controlled ovarian stimulation) procedure and pregnancy outcome, and also compare the efficiency and safety of different ascites draining ways of repeated paracentesis and central venous catheter.

\section{Materials And Methods}




\section{Patient population}

Between 2012 and 2019, Among the IVF/ICSI cycles performed at the reproductive center of Peking University third hospital, 359 patients were included in the study who were hospitalized due to severe OHSS with ascites. Those whose obstetric data could not be obtained were excluded. Among 359 patients, 223 patients received Paracentesis , 136 patients received Central venous catheter. and we also match the late-onset OHSS with the non-OHSS patient after IVF-ET by age and oocyte number, and 345 cases were strictly matched冈Figure $1 \otimes$.Abortion ratios, preterm labor ratios, and birth weights were analyzed between the two groups. The study was approved by the ethics committee of the university.

All these OHSS patients were chemical pregnant and underwent an ultrasound evaluation to check for the presence of ascites and bilateral ovarian enlargement. Blood studies including a complete blood count, blood coagulation panel and biochemical test. Urine output, weight and abdominal girth were measured. For the catheter patients: first ultrasound choose the puncture position, the hollow puncture needle was placed after local anaesthesia, and then guide wire was pulled through the puncture needle, after the skin around the puncture point was expanded, the catheter was placed under the guidance of the guide wire, and after catheter reach the right place, the guidewire was pulled out. The catheter was anchored to the skin with silk suture and a gauze dressing was then applied which changed every day to observe the skin aroud the puncture site. The catheter was then connected to a draining bag that was strapped to the patient's thigh which changed everyday. Drainage was intermittent (open the gauze in the morning, and

close at night) and the catheter was kept in place until drainage ceased and ascites had resolved completely, generally no more than one week. The total volume of fluid drained and duration of drainage were recorded. No prophylactic antibiotics were administered.

For the Paracentesis patients, also first ultrasound choose the puncture position, and then puncture needle was placed after local anaesthesia, after puncture区the inner core was drawn out, and the needle was connected to a draining tube and then a draining bag, after paracentesis the needle was drawn out and the puncture point was pasted with aseptic dressing.

\section{Controlled ovarian hyperstimulation}

During the IVF cycle, the patients were managed by a standardized clinical protocol as reported previously[9]. For ovarian stimulation, the extra-long agonist, long agonist, antagonist protocols were performed. When retrieved oocyte number $\geq 15$, or $E^{2}$ on $H C G$ day $\geq 15000 \mathrm{pmol} / L$, we choose to freeze all the embryos to prevent OHSS, Day-3 or day- 5 embryos were transferred in a fresh cycle with luteal support by vaginal or intramuscular progesterone. Serum hCG was measured 14 days after ET and was considered positive for hCG level $\geq 3.5 \mathrm{mIU} / \mathrm{ml}$. Transvaginal ultrasonography at 30 days after transfer confirmed clinical pregnancy if intrauterine gestational sac was demonstrated. The primary outcome evaluation was ongoing pregnancy, defined as the visualization of fetal cardiac activity on transvaginal ultrasound at 12 weeks' gestation or later, or live birth.

\section{Statistical analysis}


All data were stored in a computerized database. The data analysis was performed by using SPSS 22.0 software (IBM Corporation, Armonk, NY, USA). Chi-square test was used for comparison of categoric variables. the distribution of variables was tested by Shapiro-Wilks test. Student's t-test was used for variables with normal distribution whereas the Mann-Whitney $U$ test was used for data with skewed distribution, For normal distributed,results were given as mean $\pm S D$ (standard deviation), for skewed distributed data, as median $(25 \%, 75 \%)$,Significance was accepted at $P<.05$. We performed binary logistic analysis to explore the ascites fluid drainages ways on pregnancy outcomes and predict the dangerous factors of OHSS after ET, odds ratios (ORs) and their $95 \%$ confidence intervals were computed. $p<0.05$ was considered statistically significant.

\section{Results}

\section{Comparison of severe OHSS and matched control group}

For comparison of the OHSS patient and the non-OHSS IVF patients, we combine group 1 and group 2 as OHSS group, and match the control group with age and oocyte number, and the result showed there is no different in the patient weight, BMI, AFC. the long agonist protocol had bigger chance of developing OHSS(P凶0.05), and biochemical pregnancy loss rate between patient in OHSS group and control group was $0.6 \%$ and $11.1 \%$ respectively, , and the abortion rate was similar between the OHSS group and control group, but early abortion in OHSS group was much less than in control group ( P 0.007), and there was 2 cases of mid-term abortion in OHSS group, one case was 21-trisomy syndrome, and another was because of cheilopalatognathus.and there was no fetal abnormality in the control group(Table 1 and 2 ).

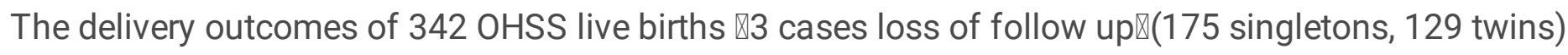

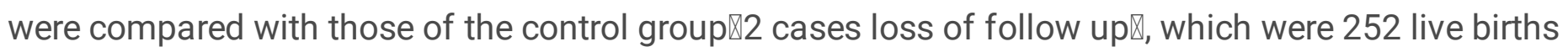
(181 singletons, 71 twins). We found significant differences in the rates of live birth (88.9\% vs. $73.5 \%$ ), multiple pregnancy (twin pregnancy) was much more in OHSS group than in control group $(57.6 \% \mathrm{vs}$ $28.1 \%$ respectively), and there was no significant difference between singleton and twin preterm rate and neonatal birth weight between OHSS and control group(Table 1 and 2)..

Binary logistic regression analysis with $p$-values less than 0.05 revealed that ovarian stimulation protocol showed relatively high odds ratio, fetal sac number (OR 2.53; $\mathrm{Cl} 1.80-3.55$ ) or long-protocol (OR , 2.72; $\mathrm{Cl}$, 1.09-6.86) showed relatively high odds ratio, and BMI and $E^{2}$ on HCG day can't predict the OHSS(Table 3)...

\section{Comparison of OHSS Group 1 and Group 2}

Demographic and cycle characteristics of patients in group 1 (once or multiple paracentesis) and group 2 (central venous catheter) with severe OHSS were compared, There was no statistically significant difference between the two groups when age, duration of infertility, and BMI were compared. 
And in the paracentesis group, only 56.6\% (126/223) patients had once paracentesis, 43.4\% (97/223) patients had multiple needle paracentesis, 2 patients had 7 times puncture (Figure 2).

The catheter was successfully placed at first attempt in all 136 patients with no complications such as bowel injury which is especially noted at the time of insertion. All the patients had immediate partial resolution of symptoms and signs following the drainage of ascitic fluid. Complete improvement of clinical symptoms usually occurred within the first two days after insertion of the Catheter. No recurrence of symptoms or signs occurred in any of the patients. In addition, with comprehensive treatment, for example, intravenous transfusion, there was immediate improvement in urine output and blood test parameters, including white blood count, haemoglobin, haematocrit levels. The catheter was kept in place for an average of $5.96 \pm 1.38$ days in group 2, and because the catheter was kept less than 7 days in case of infection, so we usually take off the catheter less than 7 days after placement, and 19 patients had paracentesis afterwards, and in group 1, the average times of paracentesis were $1.96 \pm 1.27 \mathrm{~d}$ (Table 4). Group 1drained an total volume of $4500 \mathrm{ml}$ versus $11228 \mathrm{ml}$ in group2. The difference between the two groups was statistically significant. and the hospitalization days in group 1 were $5(3 ; 8)$ versus $7(6 ; 9) d$ in group 2, because most of the patients kept the catheter for one week, and after one week, the patients had passed the peak time of late OHSS, and many of them took off the catheter and discharged from the hospital, with no fluctuation of the illness, and 18 of 131 (13.7\%) patients had paracentesis after catheter took off.

There was no reported incidence of wound or intraabdominal infection. Only some patient reported redness around the site of the wound. The catheter was removed and wound swab culture was negative for microorganisms. One patient in group 2 removed the catheter because of abdominal pain. There was no significant difference between the two groups in comparison of live birth,. the birth weight of singleton and twin between these two groups, but the premature birth rate of singleton in group 1 was $9.3 \%$ (10/107), whereas in group 2, it was 3.75\%, and the premature birth rate of twin in group 1 was $44.1 \%$ (41/93), whereas in group 2, it was $42.3 \%(22 / 52)$ (Table 6).

We performed a regression analysis to examine the independent contribution of different variables to the ongoing pregnancy rates, the result show there is no significant difference between the group 1 (once or repeated paracentesis) and group 2 (central venous catheter)

\section{Discussion}

Several strategies to prevent OHSS has been reported in the literature, including the use of low dose gonadotropins and freeze all embryos and the $\mathrm{GnRH}$ agonist as an oocyte trigger in $\mathrm{GnRH}$ antagonist cycles[10].These strategies can prevent most of the early onset OHSS, and compared with early OHSS, we pay more attention to late OHSS, because it tends to be more severe and last longer time, and patients had symptoms which maybe lethal. From the previous study, we know young age and number of oocytes retrieved are high risk factors, clinically we noticed within the same age range and oocyte number range, they all have chemical pregnancy after embryos transfer, some patients develop into OHSS and some 
patient are normal, what is the high risk factor? To investigate this problem, we matched the OHSS patient with control group by age and number of oocytes retrieved, and compare the basic data of these patients, and the result showed the less basic follicle number, GnRH antagonist cycle, singleton maybe the protective factors. Our result is in consistent with the research which reported that late OHSS was induced by the rising serum concentration of HCG produced by the early pregnancy, and associated only with multiple gestation[3].

Multiple studies have reported that the clinical pregnancy rate in OHSS patients is significantly higher than that in general IVF patients or nonखOHSS patients[11-13]. This finding is similar to our results $₫$ for the late onset OHSS, pregnancy triggers and aggravate OHSS, because endogenous HCG plays an important role in this process, these explains why in our research multiple pregnancy was much more in OHSS group than control group and the data also indicate that singleton and twin pregnancy complicated with the severe OHSS, necessitating ascites fluid drainage, are not at risk for preterm labor and IUGR.

In case of severe ascites associated with OHSS, ascites drainage is necessary, because severe ascites intra-abdominal pressure is increasing, uterine perfusion decreases, and for some OHSS patients with respiratory compromise due to massive ascites, repeated abdominal paracentesis has been proposed, and it can also improve the respiratory symptoms in patients with pleural effusion. Repeated abdominal paracentesis has the danger of infection, vascular, ovarian and gut injury, and bring more pain and inconvenience to the patient, and abdominal pressure fluctuate drastically, Following abdominal paracentesis, in our data, treatment required drainage of fluid mostly one to three times, in some cases even six to seven times, Compared with standard paracentesis, central venous catheter is a way of continuous drainage of ascetic fluid, its use improves patient care and reduces suffering, studies have reported the use of central venous drainage in the ascites effusion[14-16], but our study is the first in compare the treatment effect of standard paracentesis and central venous catheter. The findings reported herein indicate that central venous catheter is a convenient way of ascites drainage, avoiding repeated paracentesis, the patients symptoms relieve as soon as the central venous catheter was placed, as long as the catheter was in place, there was no recurrence of symptoms, the abdominal pressure was stable, according to our data the preterm rate of the central venous group was much low than the paracentesis group, when OHSS does occur, and paracentesis is necessary for severe ascites, when repeated attempts seem likely, central venous catheter is a better alternative to repeated abdominal paracentesis in the management of severe ascites of severe OHSS.

There are limitations associated with the present study, the severe OHSS patients with ascites all gain the biochemical improvement after either paracentesis or catheter placement, and we didn't compare the change of the blood test before and after the treatment, because our study is an retrospective study, the time of the blood test wasn't fixed.

In conclusion, this preliminary report suggests that for the IVF-ET patients, less basic follicle number, $\mathrm{GnRH}$ antagonist cycle, singleton maybe the protective factors for the late-onset OHSS. Paracentesis and 
central venous catheter are all the effective modality to manage the ascites for the severe OHSS patients. For the patients who have the possibility of repeated paracentesis, catheter is a safe and effective way.

\section{Abbreviation}

ovarian hyperstimulation syndrome (OHSS)

vascular endothelial growth factor (VEGF)

In vitro fertilization (IVF)

Intracytoplasmic sperm injection (ICSI)

embryo transfer (ET)

follicle-stimulating hormone (FSH)

human chorionic gonadotropin (hCG)

odds ratios (ORs)

\section{Declarations}

Ethics approval and consent to participate: This study procedure and protocol were approved by the ethics committee of Peking University Third Hospital, Peking University, and was performed in accordance with the Declaration of Helsinki

Consent for publication; Not applicable

Availability of data and material: yes

Competing interests $\otimes$ The authors declare that they have no competing interests" $\square$

Funding: The interdisciplinary medicine Seed Fund of Peking University (BMU2017MC007).

Authors' contributions: Li rong designed research and revise the manuscript, Ren yun analyzed the data and wrote the article, Yang rui and Yang shuo, Song donghong collected data, Qiao Jie revised the manuscript, All authors read and approved the final manuscript.

\section{References}


1. Rizk, B. and M. Aboulghar, Modern management of ovarian hyperstimulation syndrome. Hum Reprod, 1991. 6(8): p. 1082-7.

2. Goldsman, M.P., et al., Increased capillary permeability induced by human follicular fluid: a hypothesis for an ovarian origin of the hyperstimulation syndrome. Fertil Steril, 1995. 63(2): p. 268-72.

3. Lyons, C.A., et al., Early and late presentation of the ovarian hyperstimulation syndrome: two distinct entities with different risk factors. Hum Reprod, 1994. 9(5): p. 792-9.

4. Golan, A., et al., Ovarian hyperstimulation syndrome: an update review. Obstet Gynecol Surv, 1989. 44(6): p. 430-40.

5. Navot, D., P.A. Bergh, and N. Laufer, Ovarian hyperstimulation syndrome in novel reproductive technologies: prevention and treatment. Fertil Steril, 1992. 58(2): p. 249-61.

6. Borenstein, R., et al., Severe ovarian hyperstimulation syndrome: a reevaluated therapeutic approach. Fertil Steril, 1989. 51(5): p. 791-5.

7. Aboulghar, M.A., et al., Ultrasonically guided vaginal aspiration of ascites in the treatment of severe ovarian hyperstimulation syndrome. Fertil Steril, 1990. 53(5): p. 933-5.

8. Padilla, S.L., et al., Abdominal paracentesis for the ovarian hyperstimulation syndrome with severe pulmonary compromise. Fertil Steril, 1990. 53(2): p. 365-7.

9. Liu, N., et al., Comparison of follicular fluid amphiregulin and EGF concentrations in patients undergoing IVF with different stimulation protocols. Endocrine, 2012. 42(3): p. 708-16.

10. Youssef, M.A. and S. Mourad, Volume expanders for the prevention of ovarian hyperstimulation syndrome. Cochrane Database Syst Rev, 2016(8): p. CD001302.

11. Abramov, Y., U. Elchalal, and J.G. Schenker, Obstetric outcome of in vitro fertilized pregnancies complicated by severe ovarian hyperstimulation syndrome: a multicenter study. Fertil Steril, 1998. 70(6): p. 1070-6.

12. Raziel, A., et al., Increased early pregnancy loss in IVF patients with severe ovarian hyperstimulation syndrome. Hum Reprod, 2002. 17(1): p. 107-10.

13. Luke, B., et al., Factors associated with ovarian hyperstimulation syndrome (OHSS) and its effect on assisted reproductive technology (ART) treatment and outcome. Fertil Steril, 2010. 94(4): p. 1399-404.

14. Abuzeid, M.I., et al., Pigtail catheter for the treatment of ascites associated with ovarian hyperstimulation syndrome. Hum Reprod, 2003. 18(2): p. 370-3.

15. Al-Ramahi, M., et al., A novel approach to the treatment of ascites associated with ovarian hyperstimulation syndrome. Hum Reprod, 1997. 12(12): p. 2614-6. 
16. Abuzeid, M., et al., Outpatient Management of Severe Ovarian Hyperstimulation Syndrome (OHSS) with Placement of Pigtail Catheter. Facts Views Vis Obgyn, 2014. 6(1): p. 31-7.

\section{Tables}

Table 1: Baseline and stimulation characteristics of the late-onset OHSS group and the control group.

\begin{tabular}{|c|c|c|c|}
\hline & OHSS group『345】 & Controlø345】 & $P$ \\
\hline Weight(Kg) & $57 \rrbracket 51 \rrbracket 63 \rrbracket$ & $58 \rrbracket 52 \rrbracket 65 \rrbracket$ & 0.130 \\
\hline $\mathrm{BMI}\left(\mathrm{Kg} / \mathrm{m}^{2}\right)$ & $21.30 \rrbracket 19.53 \rrbracket 23.42 \rrbracket$ & $21.77 \rrbracket 19.87 \rrbracket 24.06 \rrbracket$ & 0.034 \\
\hline AFC & $14 \otimes 10 \otimes 17 \rrbracket$ & $11 \rrbracket 8 \rrbracket 15 \rrbracket$ & 0.000 \\
\hline Estrogen level on HCG day(pmol/L) & $11545(7133 ; 14846)$ & $9710(6371 ; 13891)$ & 0.000 \\
\hline PCOS cases & $19.7 \%(68 / 345)$ & $14.2 \%(49 / 345)$ & 0.054 \\
\hline COS protocol(Extra-long ) & $13.6 \% \otimes 47 / 298 \rrbracket$ & $12.8 \% \otimes 44 / 301 \rrbracket$ & 0.736 \\
\hline COS protocol(Antagonist ) & $32.6 \%(112 / 233)$ & $39.1 \%(135 / 210)$ & 0.072 \\
\hline COS protocol(Long ) & $50.7 \%(175 / 170)$ & $42 \%(145 / 200)$ & 0.022 \\
\hline
\end{tabular}

Table 2: Pregnancy outcomeof the late-onset OHSS group and the control group. 


\begin{tabular}{|c|c|c|c|}
\hline & $\begin{array}{l}\text { OHSS group } \\
\text { 囚345区 }\end{array}$ & Control囚345】 & $P$ \\
\hline Biochemical pregnancy loss(\%) & $0.6 \%(2 / 342)$ & $11.1 \%(38 / 343)$ & 0.000 \\
\hline Live birth rate (\%) & $88.9 \% \bowtie 304 / 342 \rrbracket$ & $73.5 \% \rrbracket 252 / 343 \rrbracket$ & 0.000 \\
\hline Twin pregnancy(\%) & $57.6 \% \bigotimes 175 / 304 \rrbracket$ & $28.1 \% \otimes 71 / 253 \rrbracket$ & 0.000 \\
\hline Ectopic pregnancy(\%) & $0(0 / 342)$ & $6.4 \%(18 / 343)$ & 0.000 \\
\hline Abortion(\%) & $9.9 \%(34 / 342)$ & $13.1 \%(45 / 343)$ & 0.193 \\
\hline Early abortion(\%) & $5.0 \%(17 / 342)$ & $10.5 \%(36 / 343)$ & 0.007 \\
\hline Late abortion(\%) & $5.0 \%(17 / 342)$ & $2.6 \%(9 / 343)$ & 0.108 \\
\hline Preterm delivery of Single pregnancy (\%) & $8.0 \%(13 / 162)$ & $4.4 \%(8 / 181)$ & 0.164 \\
\hline $\begin{array}{l}\text { Average newborn weight of single } \\
\text { pregnancy(mg) }\end{array}$ & $\begin{array}{l}3250 \rrbracket 3000 \rrbracket \\
3580 \rrbracket\end{array}$ & 3350『3040『3700』 & 0.070 \\
\hline Preterm delivery of twin pregnancy(\%) & $45 \%(58 / 129)$ & $31 \%(22 / 71)$ & 0.054 \\
\hline $\begin{array}{l}\text { Average newborn weight of twin } \\
\text { pregnancy }(\mathrm{mg})\end{array}$ & $\begin{array}{l}2550 \rrbracket 2300 \rrbracket \\
2780 \rrbracket\end{array}$ & $\begin{array}{l}2590 \rrbracket 2387.50 \rrbracket \\
2786.25 \rrbracket\end{array}$ & 0.469 \\
\hline
\end{tabular}

Table 3: logistic regression for severe OHSS

\begin{tabular}{|llll|}
\hline & OR & $95 \% \mathrm{Cl}$ & $P$ \\
\hline BMI & 0.99 & $0.96-1.02$ & 0.55 \\
\hline AFC & 1.07 & $1.04-1.10$ & 0.00 \\
\hline Extra-long & 2.63 & $0.97-7.14$ & 0.05 \\
\hline Antagonist & 1.81 & $0.71-4.62$ & 0.21 \\
\hline long & 2.72 & $1.09-6.86$ & 0.03 \\
\hline Short & & 1.0 reference & \\
\hline E2on HCG day & 1.000 & $1.00-1.00$ & 0.216 \\
\hline Multiple pregnancy & 2.53 & $1.80-3.55$ & 0.00 \\
\hline
\end{tabular}


OR: odds ratio囚Cl confidence interval

Table4 :Paracentesis times in late-onset OHSS patients with ascites

\begin{tabular}{|ll|}
\hline Patients number & Paracentesis times \\
\hline Paracentesis patients(223) & $1.96 \pm 1.27$ \\
\hline
\end{tabular}

Table5: Baseline and stimulation characteristics ofparacentesis and Catheter group in the late-onset severe OHSS with ascites

\begin{tabular}{|c|c|c|c|}
\hline & $\begin{array}{l}\text { Paracentisis group } \\
\square 223 \square\end{array}$ & Catheter group囚136》 & $P$ \\
\hline Age & $30.37 \pm 3.80$ & $30.56 \pm 3.82$ & 0.653 \\
\hline Weight(Kg) & $57.00 \rrbracket 50.75 \rrbracket 62.00 \rrbracket$ & $56.00 \rrbracket 51.00 \rrbracket 63.00 \rrbracket$ & 0.824 \\
\hline \multirow[t]{2}{*}{$\mathrm{BMI}\left(\mathrm{Kg} / \mathrm{m}^{2}\right)$} & $21.2450 \rrbracket 19.5825 \rrbracket$ & 21.4000 & 0.195 \\
\hline & $23.2575 \mathbb{Q}$ & $₫ 23.7000 \rrbracket$ & \\
\hline $\begin{array}{l}\text { Ascites draining volume during } \\
\text { hospitalization(ml) }\end{array}$ & $\begin{array}{l}4500.00 \rrbracket 3128.00 \rrbracket \\
8496.00 \rrbracket\end{array}$ & $\begin{array}{l}11228.00 \\
\bigotimes 8090.75 ; 14564.00 \rrbracket\end{array}$ & 0.000 \\
\hline Hospitalized days & $5 \rrbracket 3 \rrbracket 8 \rrbracket$ & $7 \rrbracket 6 \otimes 9 \bigotimes$ & 0.000 \\
\hline
\end{tabular}

Table 6: Pregnancy outcomeof the paracentesis and Catheter group in late-onset OHSS patients 


\begin{tabular}{|c|c|c|c|}
\hline & $\begin{array}{l}\text { Paracentesis gourp } \\
\square 223 \rrbracket\end{array}$ & $\begin{array}{l}\text { Catheter group } \\
\text { ब136凹 }\end{array}$ & $P$ \\
\hline Biochemical pregnancy loss(\%) & $0.9 \%(2 / 220)$ & $0 \%(0 / 136)$ & 0.527 \\
\hline Abortion rate(\%) & $9.1 \%(20 / 220)$ & $11.8 \%(16 / 136)$ & 0.712 \\
\hline Early abortion(\%) & $4.1 \%(9 / 220)$ & $6.6 \%(9 / 136)$ & 0.364 \\
\hline Late abortion(\%) & $4.5 \%(10 / 220)$ & $5.1 \%(7 / 136)$ & 0.796 \\
\hline Live birth number & $1.56 \pm 0.557$ & $1.52 \pm 0.514$ & 0.437 \\
\hline Live birth rate(\%) & $90 \%(198 / 220)$ & $87.5 \% \bigotimes 119 / 136 \rrbracket$ & 0.463 \\
\hline \multirow{2}{*}{$\begin{array}{l}\text { Average newborn weight of single } \\
\text { pregnancy }(\mathrm{mg})\end{array}$} & $2534.00 \rrbracket 2047.00 \rrbracket$ & 2274.50囚1907.25》 & \multirow[t]{2}{*}{0.863} \\
\hline & $3185.00 \bigotimes$ & 2622.75区 & \\
\hline \multirow{2}{*}{$\begin{array}{l}\text { Average newborn weight of twin } \\
\text { pregnancy(mg) }\end{array}$} & 2550.00』 & $2550.00 \bigotimes$ & \multirow[t]{2}{*}{0.422} \\
\hline & $2350.00 \rrbracket 2706.25 \rrbracket$ & $2275.00 \rrbracket 2887.50 \rrbracket$ & \\
\hline Preterm delivery of Single pregnancy & $(9.3 \%) 10 / 107$ & (3.75\%) 3/77 & 0.000 \\
\hline Preterm delivery of twin pregnancy & (44.1\%) 41/93 & (42.3\%) 22/52 & 0.836 \\
\hline
\end{tabular}

\section{Figures}




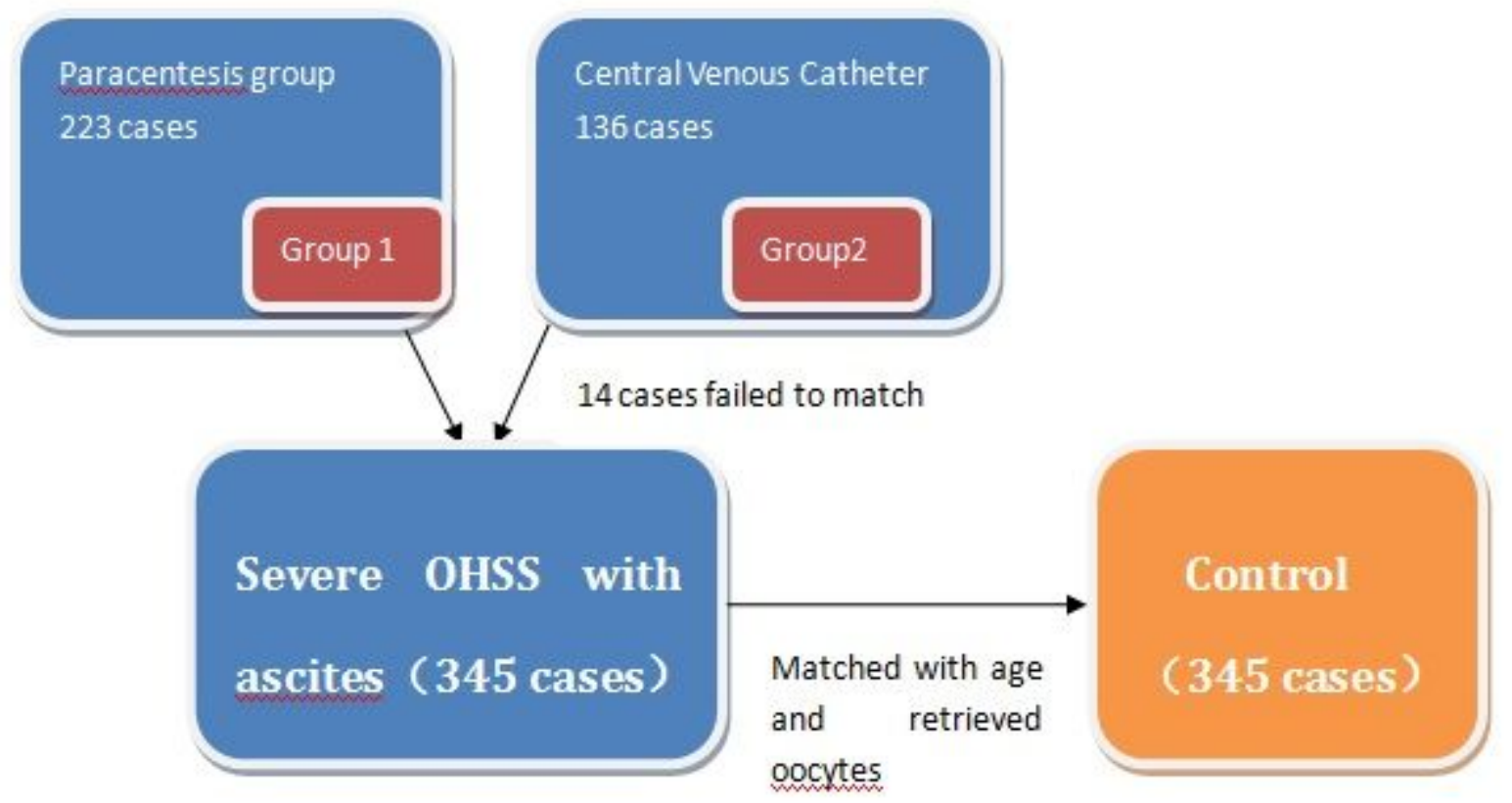

Figure 1

the groups of patients 


\begin{tabular}{|l|r|r|}
\hline rate of different paracentesis times \\
\hline \\
\hline
\end{tabular}

\section{Figure 2}

Rate of different paracentesis times in paracentesis group of late-onset OHSS patients 